\title{
Healthy Aging Alters the Functional Connectivity of Creative Cognition in the Default Mode Network and Cerebellar Network
}

\section{OPEN ACCESS}

Edited by:

Allison B. Reiss,

New York University, United States

Reviewed by:

Ling-Li Zeng,

National University of Defense Technology, China

Chu-Chung Huang,

East China Normal University, China

*Correspondence:

Deepa Madathil

deepa.m@vit.ac.in

Chih-Mao Huang

cmhuang@nctu.edu.tw

Received: 18 September 2020 Accepted: 19 January 2021 Published: 18 February 2021

Citation:

Patil AU, Madathil D and Huang C-M (2021) Healthy Aging Alters the Functional Connectivity of Creative Cognition in the Default Mode Network and Cerebellar Network. Front. Aging Neurosci. 13:607988. doi: 10.3389/fnagi.2021.607988

\begin{abstract}
Abhishek Uday Patil ${ }^{1,2}$, Deepa Madathil ${ }^{1 *}$ and Chih-Mao Huang ${ }^{2,3,4 *}$
${ }^{1}$ Department of Sensor and Biomedical Technology, School of Electronics Engineering, Vellore Institute of Technology, Vellore, India, ${ }^{2}$ Department of Biological Science and Technology, National Chiao Tung University, Hsinchu, Taiwan, ${ }^{3}$ Center for Intelligent Drug Systems and Smart Bio-Devices (IDS $\left.{ }^{2} B\right)$, National Chiao Tung University, Hsinchu, Taiwan, ${ }^{4}$ Cognitive Neuroscience Laboratory, Institute of Linguistics, Academia Sinica, Taipei, Taiwan
\end{abstract}

Creativity is a higher-order neurocognitive process that produces unusual and unique thoughts. Behavioral and neuroimaging studies of younger adults have revealed that creative performance is the product of dynamic and spontaneous processes involving multiple cognitive functions and interactions between large-scale brain networks, including the default mode network (DMN), fronto-parietal executive control network (ECN), and salience network (SN). In this resting-state functional magnetic resonance imaging ( $r s-f M R I)$ study, group independent component analysis (group-ICA) and resting state functional connectivity (RSFC) measures were applied to examine whether and how various functional connected networks of the creative brain, particularly the default-executive and cerebro-cerebellar networks, are altered with advancing age. The group-ICA approach identified 11 major brain networks across age groups that reflected age-invariant resting-state networks. Compared with older adults, younger adults exhibited more specific and widespread dorsal network and sensorimotor network connectivity within and between the DMN, fronto-parietal ECN, and visual, auditory, and cerebellar networks associated with creativity. This outcome suggests age-specific changes in the functional connected network, particularly in the default-executive and cerebro-cerebellar networks. Our connectivity data further elucidate the critical roles of the cerebellum and cerebro-cerebellar connectivity in creativity in older adults. Furthermore, our findings provide evidence supporting the default-executive coupling hypothesis of aging and novel insights into the interactions of cerebro-cerebellar networks with creative cognition in older adults, which suggest alterations in the cognitive processes of the creative aging brain.

Keywords: cognitive aging, resting-state fMRI, creative cognition, functional connectivity, group independent component analysis

\section{INTRODUCTION}

Creative thinking, defined as the iterative processes by which unusual and useful approaches to problem solving are developed, involves the dynamic integration of multiple cognitive mechanisms, including internally focused attention, proponent-response inhibitory control, and goal-directed memory retrieval (Benedek et al., 2014c, 2016; Beaty et al., 2016, 2019). The behavioral performance 
of this form of higher-level cognitive ability has been associated with individual variations in verbal and visuospatial working memory, crystallized and fluid intelligence, episodic memory, semantic knowledge, and executive function (Markman and Dietrich, 2010; Duff et al., 2013; Jauk et al., 2013; Silvia et al., 2013; Benedek et al., 2014b; Kenett et al., 2014; Mammarella et al., 2014; Addis et al., 2016). These associations suggest that various brain regions and networks that support multidimensional cognitive processes contribute to creative thinking.

Early neuroimaging studies identified associations between the neural substrates of creative cognition and a set of predominantly left-hemispheric and distributed brain regions, including the lateral prefrontal cortex (PFC), posterior parietal cortex (PPC), lateral temporal gyrus, and hippocampus (den Heuvel et al., 2010; Green et al., 2015; Kenett et al., 2015; Saggar et al., 2015; Ogawa et al., 2018; Chen et al., 2019; Wertz et al., 2020). Recent functional neuroimaging research in the field of network neuroscience used task-related and restingstate functional magnetic resonance imaging (fMRI) techniques to map the contributions of large-scale brain networks to various cognitive mechanisms associated with creativity (Bressler and Menon, 2010; Spreng et al., 2013; Beaty et al., 2016, $2018 b, 2019)$. The converging fMRI evidence from these studies identified the contributions of specific patterns of functional connectivity between the default mode network (DMN), frontoparietal executive control network (ECN), salience network (SN), visual network (VN), and lateral and medial temporal lobes to self-generated and goal-directed creative cognition processes across a range of creativity domains (Beckmann and Smith, 2005; Fox and Raichle, 2007; Fink et al., 2009, 2010, 2014; Raichle, 2010; Takeuchi et al., 2011; Abraham et al., 2012; Beaty et al., 2014, 2015, 2016, 2018a; Benedek et al., 2014a,b; Wei et al., 2014; Liu et al., 2015; Buckner and DiNicola, 2019). In a resting-state fMRI (rs-fMRI) study, Wei et al. (2014) used functional connectivity measures to investigate the critical role of the DMN in divergent thinking, and identified an association between connectivity within the DMN and the behavioral performance of divergent thinking. The authors further demonstrated that an increase in functional connectivity between the medial pre-frontal cortex and the middle temporal gyrus was associated with the processes of cognition control during divergent thinking (Wei et al., 2014). Recently, Feng et al. (2019) reported that the performance of verbal creativity was associated with a dynamic re-organization of the brain network involving various neurocognitive processes, including cognitive control, episodic memory retrieval, and imagination, and demonstrated that network interactions between the DMN and cerebellum modulate creativity in the verbal domain (Feng et al., 2019). These compelling findings support the potential contributions of large-scale brain network interactions between creativity-associated cognitive processes to individual differences in creative performance (Dietrich and Kanso, 2010; Takeuchi et al., 2012; Jung et al., 2013; Beaty et al., 2014, 2019; Wei et al., 2014).

Behavioral and neuroimaging studies conducted in younger adults have identified creative cognition as the product of dynamic and spontaneous processes involving multiple cognitive functions and large-scale brain network interactions. However, little is known about the potential effects of healthy aging on these creativity-related neurocognitive processes. Behavioral studies pertaining to age-related changes in creative cognition changes have produced conflicting evidence. A few studies have reported age-related declines in creative thought generation, whereas other studies have demonstrated that creativity performance is preserved across the human lifespan (Foos and Boone, 2008; Roskos-Ewoldsen et al., 2008; Palmiero et al., 2014; Addis et al., 2016). Critically, only two fMRI studies have examined the age-related changes in neural mechanisms and network interactions associated with creative cognition. In one pioneering study, Adnan et al. (2019b) used region of interest (ROI) functional connectivity analyses to examine age-related differences in functional brain networks during a divergent thinking task. The authors observed greater functional coupling of the neural patterns of default-executive network interactions in older subjects relative to their younger counterparts when both age groups exhibited equivalent in-scanner divergent creative performances (Adnan et al., 2019b). In the second study, Adnan et al. (2019a) used resting state functional connectivity (RSFC) measures to investigate age-related differences in the intrinsic brain network architecture associated with creative cognition. The authors found that the functional connectivity between the DMN, fronto-parietal ECN, and SN, as well as age-related default-executive network coupling, was associated with outsideof-scanner divergent thinking performance in older adults. The latter rs-fMRI study also identified the importance of the ventromedial prefrontal cortex as a network node with respect to functional connectivity in the creative aging brain (Adnan et al., 2019a). The results of both studies support the default-executive coupling hypothesis of aging (DECHA) proposed by Turner and Spreng (Turner and Spreng, 2015; Spreng and Turner, 2019), which posits that both the modulation of the prefrontal cortex and the suppression of the DMN, decrease with age. Moreover, the DECHA indicates the age-related alterations of brain connectivity associated with task demand and behavioral performance in older adults, with a greater reliance on cognitivecontrol processes which involve the prefrontal brain regions and suppression of regions of the DMN with advancing age. The DECHA further hypothesizes that there is neurocognitive flexibility between switching the use of default mode to executive process bi-directionally in younger adults. In contrast, it would become less flexible for older adults to perform the defaultexecutive coupling and would be poorly modulated by the task context.

Researchers have suggested that functional connectivity within and between the cerebellum and cerebral cortex plays a critical role in creative cognition (Ito, 2008; Neumann et al., 2018; Ogawa et al., 2018; Sunavsky and Poppenk, 2019; Sun et al., 2019). Further, several neuroanatomical and neuroimaging studies have revealed the prominent role of the cerebellum in efficient movement, coordination, and some higher cognitive functions, including creativity (Schmahmann, 1991; Ito, 1993, 2006; Vandervert et al., 2007; Buckner, 2013). For example, in an fMRI study of functional connectivity between the cerebellum and cerebral cortex in expert musicians, Pinho 
et al. (2014) demonstrated increased connectivity between the fronto-parietal network, sensorimotor network (SMN), and cerebellar network $(\mathrm{CN})$ during musical improvisation. In another fMRI study, Stoodley et al. (2012) demonstrated that neural activation patterns within and between cerebrocerebellar circuits supported cognitive tasks in various domains, including sensorimotor activity, working memory, execution control, language, visuospatial, and affective processing. These findings suggest that cerebro-cerebellar interactions are critical to the coordination of sensorimotor information, control of internally focused attention, retrieval of goal-directed memories, and production of unusual thoughts (Ramnani, 2006; Honda et al., 2018; Lin et al., 2020).

As neuroimaging studies have revealed age-related functional and structural changes in the cerebellum (Raz et al., 2005; Bernard and Seidler, 2014) and indicated the important role played by cerebro-cerebellar functional connectivity in creative thinking, we examined whether and how cerebellar interactions with other cerebral networks contribute to age-related changes in creative cognition. In this rs-fMRI study, we aimed to investigate whether and how functional connected networks associated with creativity, including the DMN, fronto-parietal ECN, SN, VN, and $\mathrm{CN}$, change with increasing age. To avoid the bias elicited by the a priori selection of brain regions from different age groups, we applied a group-independent component analysis (groupICA) approach to whole-brain RSFC at the network level. ICA is a data-driven approach in which a mathematical algorithm is used to decompose the blood oxygen level dependent (BOLD) signal into spatial and temporal independent components in rsfMRI data (McKeown et al., 1998; Beckmann and Smith, 2005; Calhoun and Adali, 2006; Cole et al., 2010; van den Heuvel and Hulshoff Pol, 2010; Bhaganagarapu et al., 2013; Rummel et al., 2013; Bi et al., 2018). This approach facilitates and improves the extraction of distinct functional connected networks. Given the unconstrained nature of the BOLD signal across younger and older participants, the estimation of group-level components in such an analysis is performed via a temporal concatenation group-ICA of data from various participants, which assumes similar spatial patterns in the brain networks across individuals (Calhoun et al., 2001; Beckmann et al., 2005; Erhardt et al., 2011; Manoliu et al., 2013). Accordingly, we then combined the groupICA and RSFC measures to examine the effects of age on creative brain connectivity.

We assessed the creative performance of individuals using the Creative Achievement Questionnaire (CAQ), an empirically valid measure of creative performance (Carson et al., 2005). The CAQ was proposed to assess achievement across 10 domains of creativity using strong psychometric parameters, and is associated with individual differences in the neuroanatomical changes of younger adults (Jung et al., 2010). Based on previous neuroimaging studies in which the modulation of the prefrontal cortex and suppression of the DMN decreased with age (i.e., DECHA) (Turner and Spreng, 2015; Spreng and Turner, 2019), and given the critical supportive role of the cerebro-cerebellar network in higher-level cognition (Ramnani, 2006; Honda et al., 2018; Lin et al., 2020), we hypothesized that healthy older adults would exhibit altered functional connectivity in areas of the DMN, ECN, and $\mathrm{CN}$ associated with creative cognition. Furthermore, we hypothesized that individual differences in creative performance would modulate the effectiveness of interactions within and between creativity-associated cerebrocerebellar networks.

\section{MATERIALS AND METHODS}

\subsection{Participants}

Twenty younger adults ( 9 females) aged $20-29$ ( $M=23.0$ years, $\mathrm{SD}=2.23)$ and 34 healthy community-dwelling older adults $(24$ females) aged 61-80 $(M=63.0$ years, $\mathrm{SD}=7.65)$ participated in this rs-fMRI study. The older participants recruited in this study comprised mainly female participants. However, a previous study on creativity failed to identify gender-related differences in creativity (Reese et al., 2001), suggesting that the number of female participants would not affect the interpretation of our results. All of the participants were screened using a detailed self-report health questionnaire, and none had a prior history of neurological (e.g., epilepsy, traumatic head injury, or other neurological disease), or psychiatric (e.g., chronic depression) disorders. All of the participants were right-handed and exhibited normal or corrected-to-normal visual acuity during screening, and none used a hearing aid. All of the participants provided written informed consent prior to participation. The study protocol was approved by the Institutional Review Board of National Chiao Tung University, Taiwan.

\subsection{Neuropsychological Assessment}

Before undergoing MRI scanning, each participant completed the Mini-Mental State Examination (MMSE) (Folstein et al., 1975), which was designed to assess global cognitive abilities such as orientation in time and space, attention and calculation, memory, and language. We also applied a battery of neuropsychological tests from the third version of the Wechsler Adult Intelligence Scale (Wechsler, 1997a) to assess age-related and individual differences in neurocognitive function, including digit symbol coding, symbol searching, block design, picture completion, matrix reasoning, arithmetic, letter-number sequencing, forward and backward digit span, vocabulary, and similarity tests. We also applied the forward and backward spatial span, immediate and delayed facial recognition, and visual reproduction tests from the third version of the Wechsler Memory Scale (Wechsler, 1997b).

\subsection{Creative Ability Assessment}

All of the participants were instructed to complete the creative achievement questionnaire (CAQ) (Carson et al., 2005) as a measure of age-related and individual differences in creativity abilities. The CAQ is a self-reported and empirically valid measure of creative performance in which strong psychometric parameters are used to assess achievement across 10 domains of creativity: arts, music, dance, architecture, creative writing, humor, invention, scientific discovery, drama, and cooking. The CAQ score indicates whether a person's creative achievements are observable and recognized or appreciated by the public in various creativity-related domains. Following a previous behavioral study in which the CAQ score distribution appeared to be highly 
skewed (Silvia et al., 2009), we standardized the CAQ scores across all of the participants prior to further analysis.

\subsection{Functional MRI Data Acquisition and Preprocessing}

All of the functional and anatomical imaging was performed on a 3T Siemens MRI scanner (Magnetron Trio, Siemens, Germany) at National Yang-Ming University, Taipei, Taiwan. The participants were asked to rest with their eyes closed for $5 \mathrm{~min}$ during the functional imaging data acquisition.

During each session, functional images were obtained using a single shot $\mathrm{T} 2{ }^{\star}$-weighted gradient echo-planer image (EPI) sequence [response time (TR)/echo time (TE) $=2,000 / 30$ $\mathrm{ms}$; flip angle $=90^{\circ} \mathrm{]}$. Thirty-three contiguous axial slices were acquired, each with a slice thickness of $4 \mathrm{~mm}$, a matrix of $64 \times 64$, and an in-plane resolution of $3.1 \times$ $3.1 \mathrm{~mm}$. Anatomical images were obtained using isotropic T1-weighted, three-dimensional (3D), ultrafast magnetizationprepared rapid acquisition with a gradient echo (MPRAGE) sequence [TR/inversion time (TI)/TE: 3,500/1,100/3.5 ms; flip angle $=7^{\circ}$ ]. One hundred and ninety-two slices with a slice thickness of $1 \mathrm{~mm}$ and field of view of 256 $\mathrm{mm}^{2}$ were acquired. All of the anatomical images were verified visually by MRI experts, and no brain abnormalities were observed.

All of the preprocessing and denoising of the acquired fMRI data were performed using the CONN toolbox (http://www.nitrc. org/projects/conn) based on MATLAB version 2019b (https:// in.mathworks.com). The preprocessing of the resting-state fMRI data was performed using the standardized pre-processing pipeline in CONN for volume-based analysis (WhitfieldGabrieli and Nieto-Castanon, 2012). The preprocessing included slice-timing correction, motion correction, realignment, and normalization. The functional data of each participant were initially realigned and unwrapped for motion estimation and correction. After re-alignment, the data were assessed and corrected for head motion artifacts. Artifact reduction tool (ART) based scrubbing was used to detect and repair bad volumes, which were defined using two measures: (1) a framewise displacement $>0.9 \mathrm{~mm}$ in all directions and (2) a global mean intensity threshold $>5$ standard deviations from the mean intensity for the entire scan.

The functional and anatomical images were normalized separately in a standardized Montreal Neurological Institute (MNI) space and then segmented into white matter (WM), gray matter (GM), and cerebrospinal fluid (CSF) using the unified segmentation and normalization procedure (Ashburner and Friston, 2005) in SPM12 (The Wellcome Center for Human Neuroimaging, London, UK, http://www.fil.ion.ucl.ac. $\mathrm{uk} / \mathrm{spm} /$ ). The normalized images were then smoothed by spatial convolution with an 8-mm full-width half-maximum Gaussian kernel (FWHM). Finally, the fMRI data were bandpass-filtered between 0.008 and $0.09 \mathrm{~Hz}$. The CompCor method in CONN (Behzadi et al., 2007) was also applied to interpret various principal components associated with the CSF and WM. The realignment parameters, CSF, and WM were used as confounding factors in the first-level resting state analysis (Behzadi et al., 2007).

\subsection{Resting-State fMRI Data Analysis}

The rs-fMRI data were subjected to group-ICA and assessed using functional connectivity measures.

\subsubsection{Group Independent Component Analysis (Group-ICA)}

The group-ICA was performed using the FastICA method for the estimation of independent spatial components and groupICA backpropagation. For this analysis, the default settings in the CONN toolbox for group-ICA were used (https://web.conntoolbox.org/; Whitfield-Gabrieli and Nieto-Castanon, 2012). The ICA maps in our work represent measures of different restingstate brain networks and connectivity at each voxel. The approach of ICA has been widely used to identify various brain networks in resting state fMRI data. The fMRI data was processed subject-wise and the group-ICA was implemented in CONN. The group level independent component activation maps in the ICA analysis are based on group-ICA methodology used by Calhoun et al. (2001), with subject level dimension reduction, concatenation across subjects and dimensionality reduction by using group-level singular value decomposition using a fastICA algorithm. The number of estimated components was set at 30 , and the dimensionality reduction factor was set at 64 . The selection of the components identified in the ICA was based on the suggestions from the previous works which used 30 components for analyses (Calhoun et al., 2001; Li et al., 2007; Abou-Elseoud et al., 2010; Abou Elseoud et al., 2011). Furthermore, a correlational spatial match-to-template in CONN was applied to identify resting state networks (RSNs) within each component.

\subsubsection{Resting State Functional Connectivity (RSFC) Measures}

In this study, the resting-state functional connectivity measures were based on the ROI-to-ROI analysis and seed-based connectivity measures defined in the CONN toolbox (https:// web.conn-toolbox.org/).

The ROI-to-ROI analysis represents the level of functional connectivity between each pair of ROIs. The functional connectivity measures were applied to the 132 default nodes defined in the CONN atlas, which normally combines the automated anatomical labeling (AAL) atlas of 26 cerebellar regions (Tzourio-Mazoyer et al., 2002) with the FSL-based Harvard-Oxford cortical and subcortical regions. Functional connectivity measures were assessed for the resting state data of each subject, and beta values representing the averagedFischer transformed pairwise correlations of specified contrast were obtained for every ROI and every subject. The Student's $t$ test was used to obtain the significant statistics between these beta values and the CAQ score.

The seed based connectivity measure was based on characterizing connectivity patterns with the set of in prior seed ROIs. Moreover, the seed-based connectivity maps were obtained with the $4 \mathrm{DMN}$ seeds and 26 seeds in the cerebellum 
defined in the CONN atlas to understand potential connectivity paths within the DMN and estimating cerebro-cerebellar connectivity measures associated with creative cognition.

The significance threshold for both functional connectivity measure and voxel-based measure was set to uncorrected voxelwise $p<0.001$, with a false discovery rate (FDR)-corrected cluster-wise $p<0.05$ (Chai et al., 2014; Geissmann et al., 2018).

\section{RESULTS}

\subsection{Demographic and Behavioral Characteristics}

All of the participants received a minimum score of 26 in the MMSE (Folstein et al., 1975), with mean scores of 29.3 and 28.7 for the younger and older participants, respectively. The older participants had fewer years of education than the younger participants $(M=12.79$ years, $\mathrm{SD}=3.29$ and $M=15.45$ years, $\mathrm{SD}=1.50$, respectively, $p=0.01$ ). The two groups had equivalent verbal ability levels, as determined using the vocabulary test and similarity test. Regarding neuropsychological parameters, we observed inter-group differences in the processing speed (digit symbol coding test, $p<0.01$; symbol search test, $p<0.01$ ), visuospatial ability (block design test, $p<0.01$; picture completion test. $p<0.05$ ), fluid intelligence (matrix reasoning test, $p<0.01$; arithmetic test, $p<0.05$ ), working memory (letternumber sequencing, $p<.01$; digit span forward, $p<0.05$; digit span backward, $p<0.01$ ), and both immediate and delayed recall measures (visual reproduction, $p<0.01$; immediate face recognition, $p<0.01$; delayed face recognition, $p<0.01$ ). This pattern, in which crystallized intelligence (i.e., vocabulary test) is spared while fluid intelligence (i.e., speed, executive function, and working memory) decreases with age, is typical of most samples in the literature on normal cognitive aging (Park et al., 2002; Huang et al., 2012; Fan et al., 2019). The demographics of both groups are depicted in Table 1.

\subsection{Resting-State fMRIs}

All of the rs-fMRI data were analyzed using a voxel threshold $p<0.001$ and an FDR-corrected cluster threshold $p<0.05$. The resting-state brain activation maps of the default-mode networks in the younger and older participants are presented in Figure 1.

\subsubsection{Group ICA Results}

In this study, the group-ICA using CONN was used to identify various brain networks in resting-state fMRI data to examine age-related alterations in the functional connectivity of creative cognition. In the ICA analysis, we calculated 30 independent components with a default $z$-value threshold of 2 to identify the brain networks associated with each ICA component. The threshold of 2 is well-defined and used to characterize connections within each component in several previous restingstate fMRI studies. Eleven resting-state brain networks were obtained using this ICA approach, including the DMN, ECN, $\mathrm{CN}, \mathrm{VN}, \mathrm{SN}$, language network (LN), fronto-parietal network (FPN), auditory network (AN), and SMR using the correlation based spatial match-to-template in CONN (Kornelsen et al.,
TABLE 1 | Demographics and Neuro-psychological results of younger and older participants.

\begin{tabular}{llcc}
\hline & Assessment used & \multicolumn{2}{c}{ Groups } \\
\cline { 3 - 4 } & & Older adults & Younger adults \\
\hline Total & & 34 & 20 \\
Age [mean (SD)] & - & $63.0(7.65) \mathrm{yrs}$. & $23.0(2.23) \mathrm{yrs}$. \\
Education [mean & & $12.79(3.29) \mathrm{yrs}$. & $15.45(1.5) \mathrm{yrs}$. \\
(SD)] & & & \\
Cognitive test & MoCA score & $24.38(6.76)$ & $28.95(1.79)$ \\
scores & [mean (SD)] & & \\
& MMSE score & $28.70(1.08)$ & $29.3(0.80)$ \\
Creativity scores & [mean (SD)] & & \\
Memory scores & CAQ [mean (SD)] & $6.91(21.82)$ & $10.65(8.76)$ \\
& Digit-span & $13.64(1.88)$ & $15.45(0.68)$ \\
& $\begin{array}{l}\text { Forward [mean } \\
\text { (SD)] }\end{array}$ & & \\
& Digit-Span & $7.25(2.23)$ & $11.8(2.54)$ \\
& $\begin{array}{l}\text { Backward [mean } \\
\text { (SD)] }\end{array}$ & & \\
& Letter-Number & $9.9(5.21)$ & $16.45(3.61)$ \\
& sequencing [mean & & \\
& (SD)] & &
\end{tabular}

2020). The major brain networks identified in both younger and older participants by the group-ICA are presented in Figure 2.

Table 2 presents the clustered areas of significant activation in the younger and older participants as identified by the groupICA. No significant differences between the older and younger adults were observed in the clustered areas of activation in the DMN. In the ECN, the older adults exhibited clustered activity in the left inferior frontal gyrus, left superior parietal gyrus (SPG), and various right hemispheric regions, including the hippocampus, lingual regions, and middle frontal regions. In contrast, in the ECN of the younger adults, bilateral activity was observed in the middle temporal gyrus (MTG) and frontal gyrus (MFG). Activity was also observed in the supplementary motor area (SMA), cerebellar regions, PCC, precuneus, and left superior frontal gyrus (SFG) in younger adults.

In the $\mathrm{CN}$, the older participants exhibited activation in the cerebellum and the anterior and middle cingulate cortex. Furthermore, the older adults exhibited activity in the precentral gyrus, insula, and supplementary motor area. In contrast, the younger adults exhibited activity in the inferior and superior frontal gyrus (IFG and SFG), putamen, and fusiform. The latter group also exhibited greater activity in the frontal regions of the brain relative to the cerebellar and temporal-occipital regions. The cerebellar cluster identified using the group-ICA is depicted in Table 4.

\subsubsection{RSFC of the Creative Brain in Younger and Older Adults}

ROI-to-ROI analysis was conducted to examine whether and how individual differences in creative ability, as measured by CAQ scores (controlled by individual differences in educational level), influenced the functional connected networks in younger and 


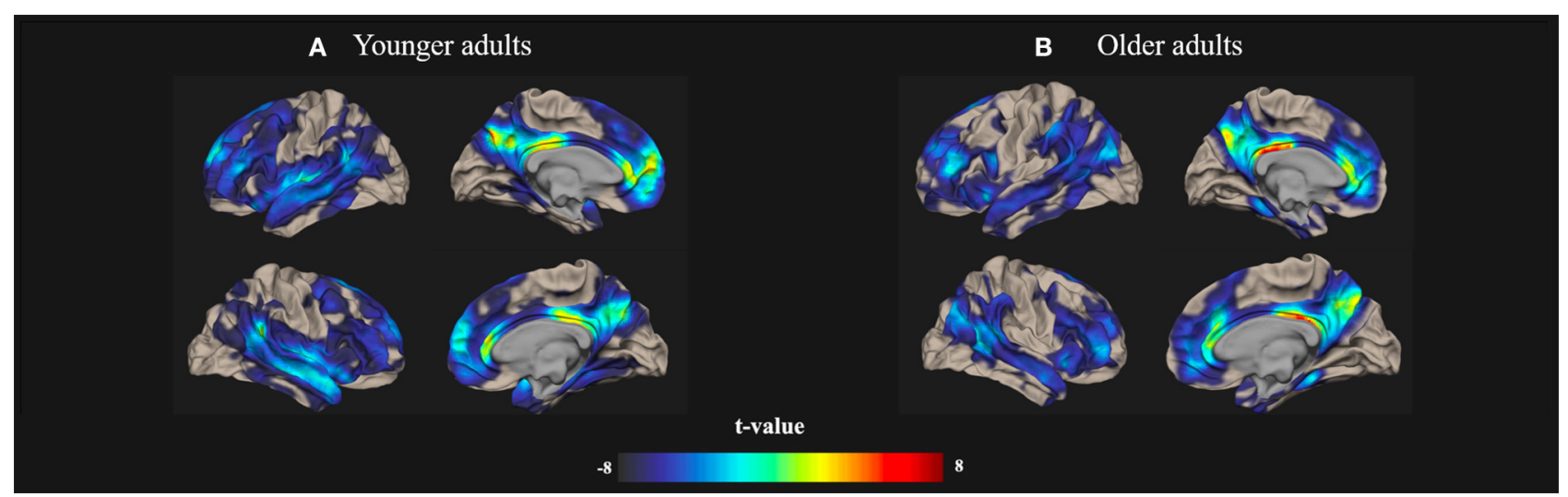

FIGURE 1 | Resting-state Default-mode brain activation associated with creativity in younger and older adults. Resting-state brain activation maps depict the default-mode networks in (A) younger and (B) older adults. A significance level of voxel-level threshold $p<0.001$ and a cluster-level threshold false discovery rate (FDR)-corrected $p<0.05$ were applied.

(a) YOUNGER ADULTS

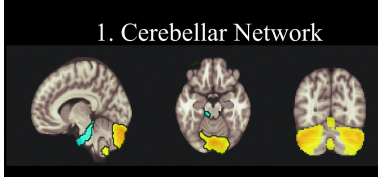

2. Salience Network

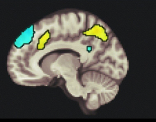

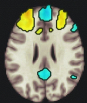
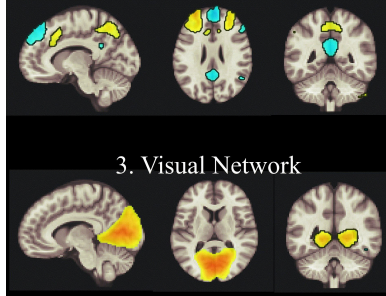

4. Sensorimotor Network
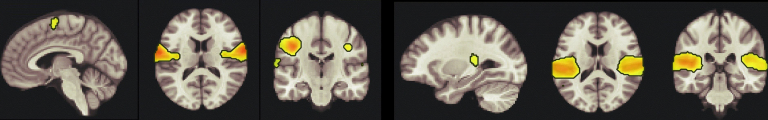

5. Language Network
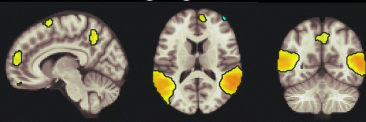

10. Executive Network (anterior)
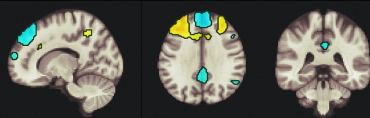

11. Medial Temporal

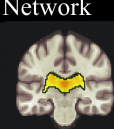

(b) OLDER ADULTS

1. Cerebellar Network

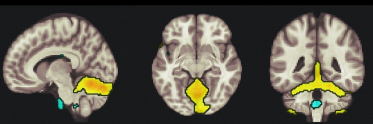

2. Salience Network

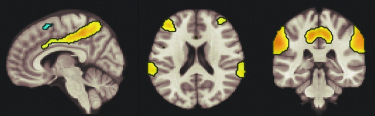

3. Visual Network

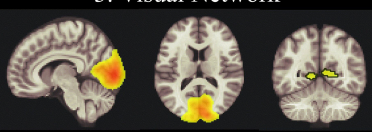

4. Sensorimotor Network

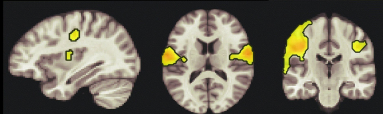

5. Language Network

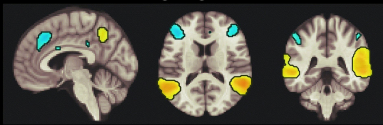

6. Default Mode Network

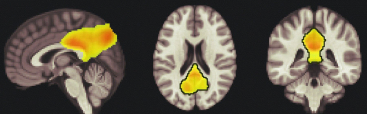


TABLE 2 | Clustered areas of significant activation during creative aging in older and younger adults identified using group independent component analysis.

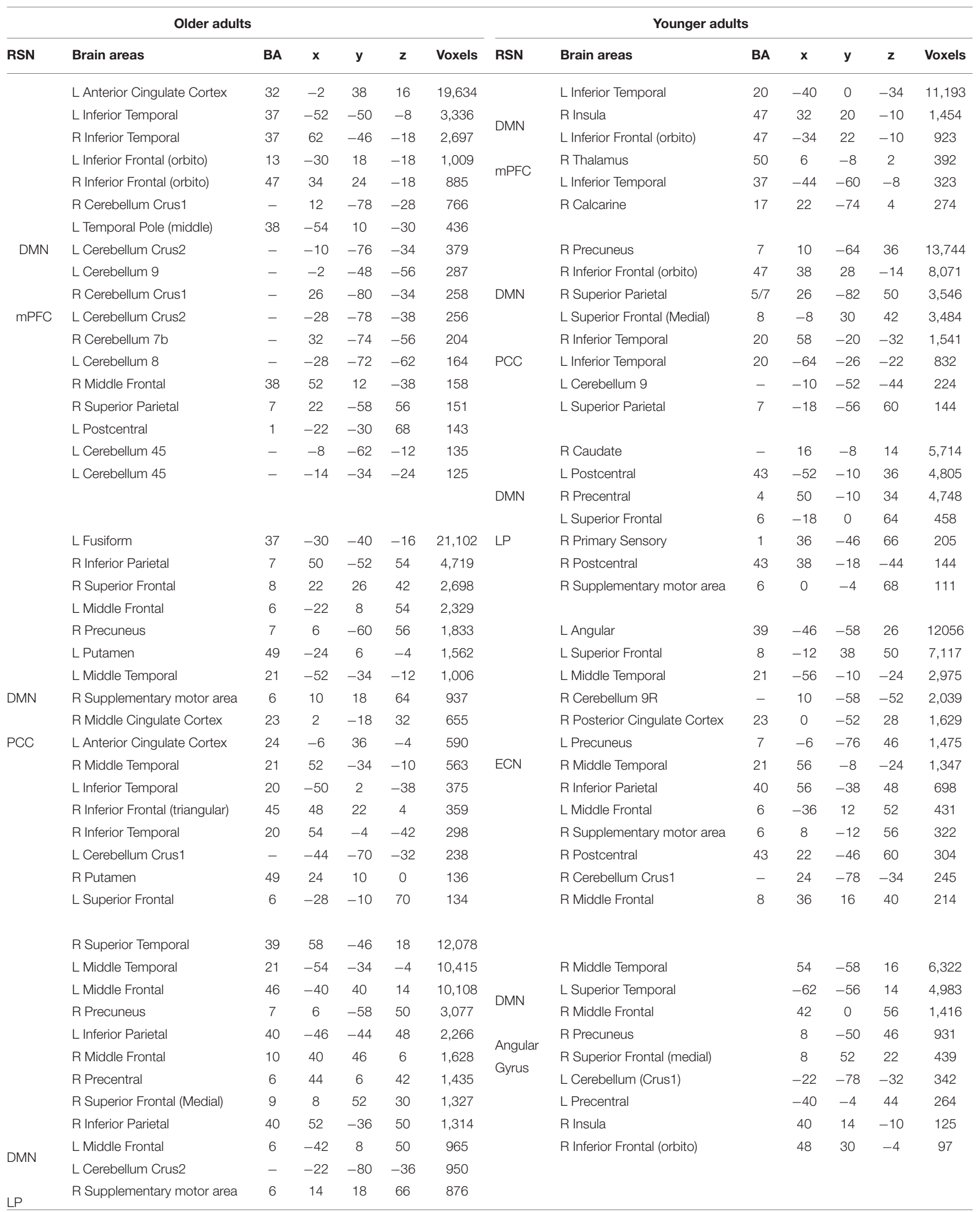


TABLE 2 | Continued

\begin{tabular}{|c|c|c|c|c|c|c|c|c|c|c|c|c|c|}
\hline \multicolumn{7}{|c|}{ Older adults } & \multicolumn{7}{|c|}{ Younger adults } \\
\hline & R Rectus & 11 & 4 & 48 & -24 & 614 & & & & & & & \\
\hline & R Cerebellum Crus2 & NA & 22 & -76 & -36 & 570 & & & & & & & \\
\hline & L Middle Cingulate Cortex & 23 & -2 & -34 & 36 & 553 & & & & & & & \\
\hline & L Cerebellum 9 & - & -4 & -50 & -52 & 525 & & & & & & & \\
\hline & R Inferior Temporal & 37 & 52 & -52 & -16 & 514 & & & & & & & \\
\hline & L Hippocampus & 30 & -18 & -36 & 2 & 167 & & & & & & & \\
\hline & L Cerebellum Crus2 & - & -42 & -62 & -42 & 119 & & & & & & & \\
\hline & L Superior Frontal & 6 & -16 & -10 & 76 & 116 & & & & & & & \\
\hline & R Para Hippocampal & 34 & 26 & -40 & -4 & 210 & & & & & & & \\
\hline & L Superior Parietal & 7 & -20 & -56 & 72 & 186 & & & & & & & \\
\hline
\end{tabular}

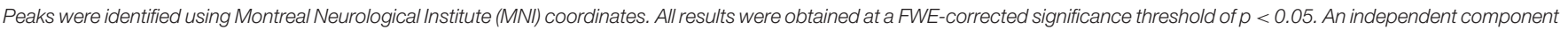

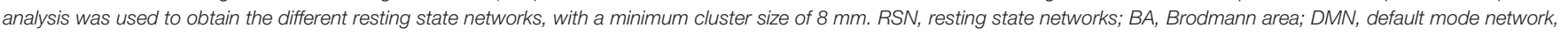

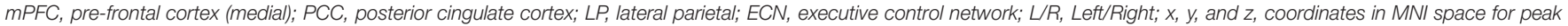
voxels in activated regions; Vol, voxels in the peak activated regions.

TABLE 3 | Effect of CAQ scores on resting-state functional connectivity in younger adults.

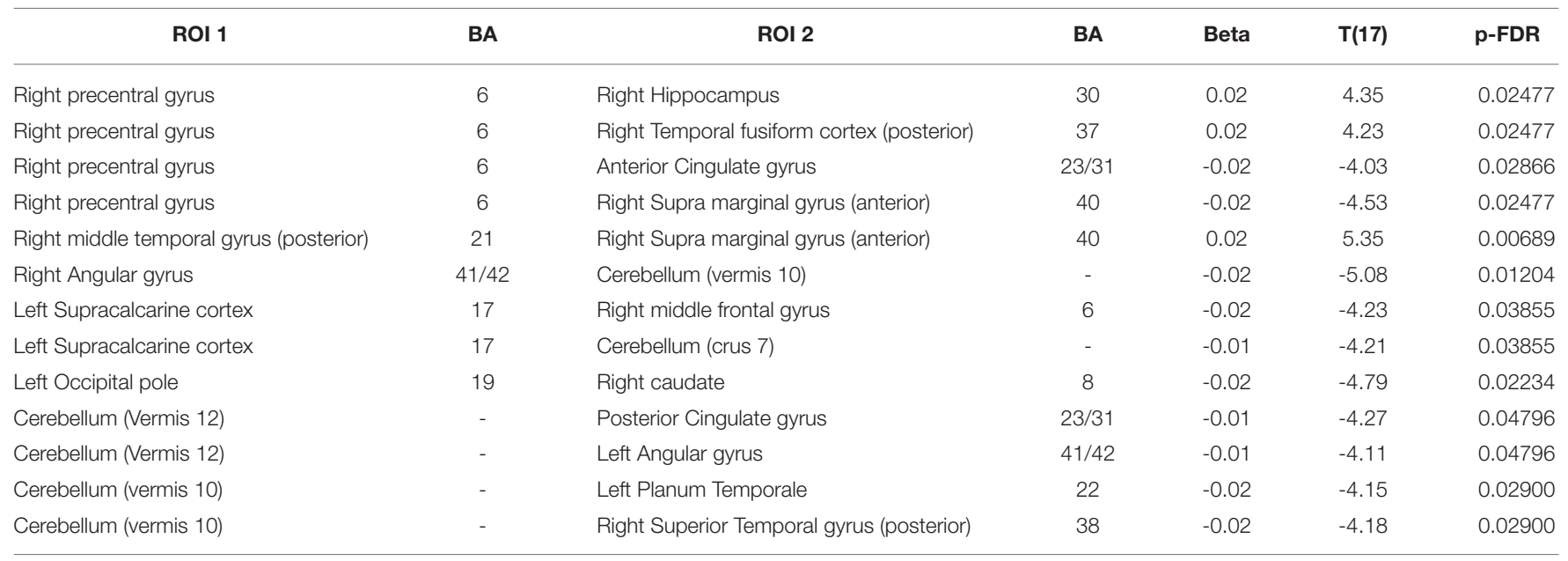

$C A Q$, Creative Achievement Questionnaire; ROI, region of interest; $B A$, Brodmann area; $p$-FDR, false discovery rate-corrected p-value.

older adults. The results of the age-related functional connectivity within and between various brain networks associated with creative performance are presented in Table 3. The effect of creative performance, measured by CAQ on resting-state functional connectivity is depicted with $3 \mathrm{D}$-Glass brain in Figure 3A and Connectogram in Figure 3B.

In younger adults, when the right medial temporal gyrus within the DMN (coordinates: $x=61, y=-22, z=-12$ ) was selected as a seed ROI, higher connectivity with the right supra marginal gyrus $(t=5.35$; p-FDR $=0.0068)$. When ROIs in the right angular gyrus were used as the seed ROIs $(x=$ 51, $y=-51, z=32$ ), lower connectivity with the cerebellum was observed $(t=-5.08 ; \mathrm{p}-\mathrm{FDR}=0.0120)$. When ROIs in the right precentral gyrus were used as a seed $(x=34, y=$ $-10, z=50$ ) higher connectivity with the right hippocampus $(t=4.35 ; \mathrm{p}-\mathrm{FDR}=0.0247)$ and right temporal fusiform cortex $(t=4.23$; p-FDR $=0.0247)$ whereas lower connectivity with the anterior cingulate cortex $(t=-4.03$; p-FDR $=0.0286)$ and right supra marginal gyrus $(t=-4.53$; $\mathrm{p}$-FDR $=0.0286)$ were observed. Within the VN, the left occipital pole exhibited lower 
connectivity with the right caudate $(t=-4.79$; $\mathrm{p}-\mathrm{FDR}=0.0223)$, the left supra calcarine cortex exhibited lower connectivity with the right middle frontal gyrus $(t=-4.23$; p-FDR $=0.0385)$ and the cerebellum $(t=-4.21$; p-FDR $=0.0385)$. Within the $\mathrm{CN}$, when the cerebellum was used as the seed ROI $(x=33, y=-63, z$ $=-48$ ), lower connectivity exhibited with the posterior cingulate gyrus $(t=-4.27$; $\mathrm{p}-\mathrm{FDR}=0.0479)$, left angular gyrus $(\mathrm{t}=-4.11$; $\mathrm{p}$-FDR $=0.0479)$, left planum temporale $(t=-4.15$; $\mathrm{p}$-FDR $=$ $0.0290)$ and right superior temporal gyrus $(t=-4.18$; p-FDR $=$ 0.0290 ). In contrast, the older adults exhibited no connectivity with the effect of CAQ when the ROI-to-ROI analysis was applied. The results have been depicted in Table 3.

Regarding network-specific interactions affected by CAQ scores, we observed greater functional connectivity between

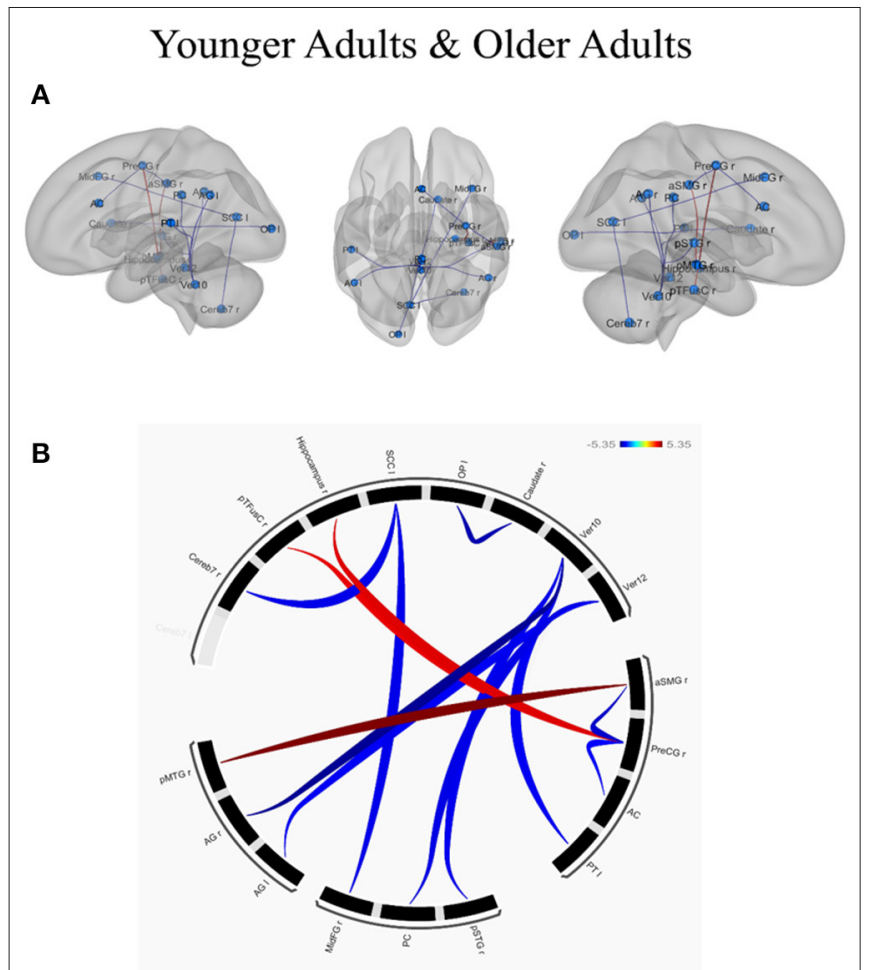

FIGURE 3 | Effect of creative performance (measured by Creative Achievement Questionnaire, $\mathrm{CAQ}$ ) on resting-state functional connectivity represented using (A) 3D-Glass brain and (B) Connectogram. (A) 3D-Glass brain: Each node is represented as a sphere (in cyan color); the positive and negative connections are represented in red and blue color, respectively. (B) Connectogram: Each node is coded with black color; the positive and negative connections are represented in red and blue color, respectively. The results were thresholded using a false discovery rate (FDR)-corrected $p<0.05$ for multiple testing correction (corrected by individual differences in educational level). OP I, left occipital pole; Caudate r, right caudate; Ver12, vermis lobule 12; Ver10, vermis lobule 10; aSMG r, right supramarginal gyrus (anterior); PreCG r, left precentral gyrus; AC, anterior cingulate cortex; PT I, left planum temporale; pSTGr, right superior temporal gyrus (posterior); PC, posterior cingulate cortex; MidFGr, right middle frontal gyrus; AG I, left angular gyrus; AG $r$, right angular gyrus; pMTGr, right middle temporal gyrus (posterior); Cereb 7r, right cerebellum lobule 7; pTFusC $r$, right temporal fusiform cortex (posterior); Hippocampus r, right hippocampus; SCC I, left supra-calcarine cortex. the SMN and the dorsal attention network $(t=-4.30$; $\mathrm{p}$ FDR $<0.05)$ in the younger adults, but did not observe this effect of CAQ scores on connectivity in the older adults. Regarding cerebro-cerebellar interactions, the anterior $\mathrm{CN}$ exhibited connectivity with the dorsal attention network ( $T=$ 2.66; $p<0.05$ ) and the posterior $\mathrm{CN}$ exhibited connectivity with the VN $(T=-2.16 ; p<0.05)$ in the younger adults. In the older adults, the anterior $\mathrm{CN}$ exhibited connections with the DMN $(T=3.20 ; p<0.05)$ and $\mathrm{SN}(T=-2.35 ; p<0.05)$, whereas the posterior $\mathrm{CN}$ exhibited connections with the dorsal attention network $(T=-2.63 ; p<0.05)$.

The voxel-wise analysis was performed on the cerebellar regions to understand the functional connections between the cerebellum and the cerebral regions during creative cognition processes. The significant clustered cerebellar areas in younger adults are presented in Table 4 . The older adults did not show any functional connections from the cerebellum to the cerebral regions which correlated with the CAQ score.

Regarding region-specific interactions within the cerebellum affected by the CAQ scores, the results of the functional

TABLE 4 | Resting-state correlations with clusters throughout the brain in younger adults with 26 cerebellar seed ROls.

\begin{tabular}{|c|c|c|c|c|c|c|}
\hline Seed ROI & Brain areas & BA & $\mathbf{x}$ & y & $\mathbf{z}$ & voxels \\
\hline \multicolumn{7}{|c|}{ YOUNGER ADULTS } \\
\hline $\begin{array}{l}\text { R Cerebellum } \\
\text { (45) }\end{array}$ & Left cingulate gyrus & 23 & -6 & -48 & 34 & 100 \\
\hline \multirow[t]{3}{*}{ R Cerebellum (7) } & Right lingual gyrus & - & 16 & -88 & 0 & 558 \\
\hline & Left Insula & 13 & -40 & -2 & 14 & 127 \\
\hline & Right precentral gyrus & 1 & 56 & -12 & 34 & 95 \\
\hline L Cerebellum (8) & Right precentral gyrus & 6 & 50 & -2 & 34 & 161 \\
\hline \multirow[t]{2}{*}{ R Cerebellum (9) } & Left middle frontal gyrus & 6 & -34 & 6 & 58 & 208 \\
\hline & Left lentiform nucleus & - & -12 & 0 & -2 & 204 \\
\hline \multirow{2}{*}{$\begin{array}{l}\text { R Cerebellum } \\
\text { (10) }\end{array}$} & Left lingual gyrus & 18 & -6 & -82 & -8 & 274 \\
\hline & Left middle occipital gyrus & 19 & -34 & -86 & 16 & 88 \\
\hline \multirow[t]{7}{*}{ Vermis (12) } & Left cingulate gyrus & 23 & -12 & -44 & 28 & 817 \\
\hline & Left medial frontal gyrus & - & -16 & 52 & 12 & 607 \\
\hline & Right Inferior parietal lobule & 39 & 46 & -60 & 38 & 416 \\
\hline & Right medial frontal gyrus & 10 & 6 & 50 & -8 & 272 \\
\hline & Left superior frontal gyrus & 8 & -10 & 34 & 52 & 142 \\
\hline & Right superior frontal gyrus & 6 & 20 & 26 & 60 & 119 \\
\hline & Left middle frontal gyrus & 8 & -34 & 18 & 46 & 91 \\
\hline \multirow[t]{2}{*}{ Vermis (3) } & Left supramarginal gyrus & - & -64 & -48 & 32 & 126 \\
\hline & Right medial frontal gyrus & 9 & 8 & 48 & 22 & 94 \\
\hline Vermis (9) & Left parahippocampal gyrus & 37 & -22 & -42 & -10 & 127 \\
\hline \multirow[t]{2}{*}{ Vermis (10) } & Right Inferior parietal lobule & 40 & 54 & -42 & 22 & 278 \\
\hline & Left superior temporal gyrus & 22 & -62 & -44 & 14 & 89 \\
\hline
\end{tabular}

Peaks were identified using the MNI coordinates. All results were obtained with the threshold significance set to an uncorrected $p<0.001$ at a voxel-wise level, with the false discovery rate (FDR)-corrected $p<0.05$ at the cluster-wise level. L/R, Left/Right; $X, Y$, and Z, coordinates in Montreal Neurological Institute (MNI) space for peak voxels in activated regions; $\mathrm{Vol}$, voxels in peak activated regions; seed $\mathrm{RO}$, seed region of interest; $B A$, Brodmann area. 
connectivity analysis revealed that younger adults showed positive correlations between the CAQ score and higher functional connectivity within the cerebellum. In older adults, however, showed positive correlations between the CAQ score and lower functional connectivity in the cerebellum. The restingstate positive and negative correlations within the cerebellum in younger and older adults are depicted in Table 5.

\section{DISCUSSION}

Using a group-ICA and RSFC approach, this rs-fMRI study identified age-related differences in functional connectivity within the default-executive and cerebro-cerebellar network interactions associated with creativity. The group-ICA approach identified 11 major brain networks across age groups, which reflected the age-invariant resting-state networks. Compared with the older participants, the younger participants exhibited more specific and widespread dorsal network and SMN connectivity within and between the $\mathrm{DMN}, \mathrm{VN}, \mathrm{AN}$, frontoparietal network, and $\mathrm{CN}$. These observations suggest the occurrence of age-specific changes in the functional connected network, particularly in the default-executive network and cerebro-cerebellar network. In the younger adults, the connections identified between the cerebellum and paracingulate cortex identified regions critical to pre-potent response inhibition that were not seen in older adults. Our connectivity data further elucidate the critical roles of the cerebellum and cerebro-cerebellar connectivity (particularly the DMN, frontoparietal network, VN, SN, and dorsal attention network) in the creative brain with age. Further, our findings are consistent with the DECHA and provide novel evidence supporting cerebrocerebellar network interactions related to creative cognition in older adults. Taken together, our results suggest changes to creative cognitive processes in the aging brain.

Several studies have explored the potential link of RSFC between various brain networks with creative cognition. A functional link has been identified between creative cognition and the DMN, especially the PCC, medial prefrontal cortex, and lateral parietal regions (Takeuchi et al., 2011; Beaty et al., 2015, 2018a). Behavioral measures have been used to link restingstate connections between the DMN and ECN with creative cognition (Beaty et al., 2018b). In this rs-fMRI study, we observed significant positive connections with DMN regions and other DMN and posterior regions in the older adults, and these positive connections were correlated significantly with the creative cognition scores. In the younger adults, the DMN exhibited significant positive connections only with frontal brain regions. The more negative connectivity in older adults relative to their younger counterparts suggests the existence of agerelated changes in functional connected networks within and between the DMN. Similarly, when seed ECN regions were used, significant connections with the posterior and frontal regions were observed in the older adults, whereas only connections with the frontal regions were observed in the younger adults. When seed VN (i.e., temporo-occipital regions) regions were used, both the older and the younger adults exhibited positive significant connections with the creative cognition scores. Our observations of functional connectivity in the DMN and ECN are consistent with the DECHA, and studies related to that hypothesis also observed a beneficial shift in the neural architecture related to creative cognition in older adults (Adnan et al., 2019a,b).

TABLE 5 | Resting-state positive and negative correlations within the cerebellum in younger adults and older adults.

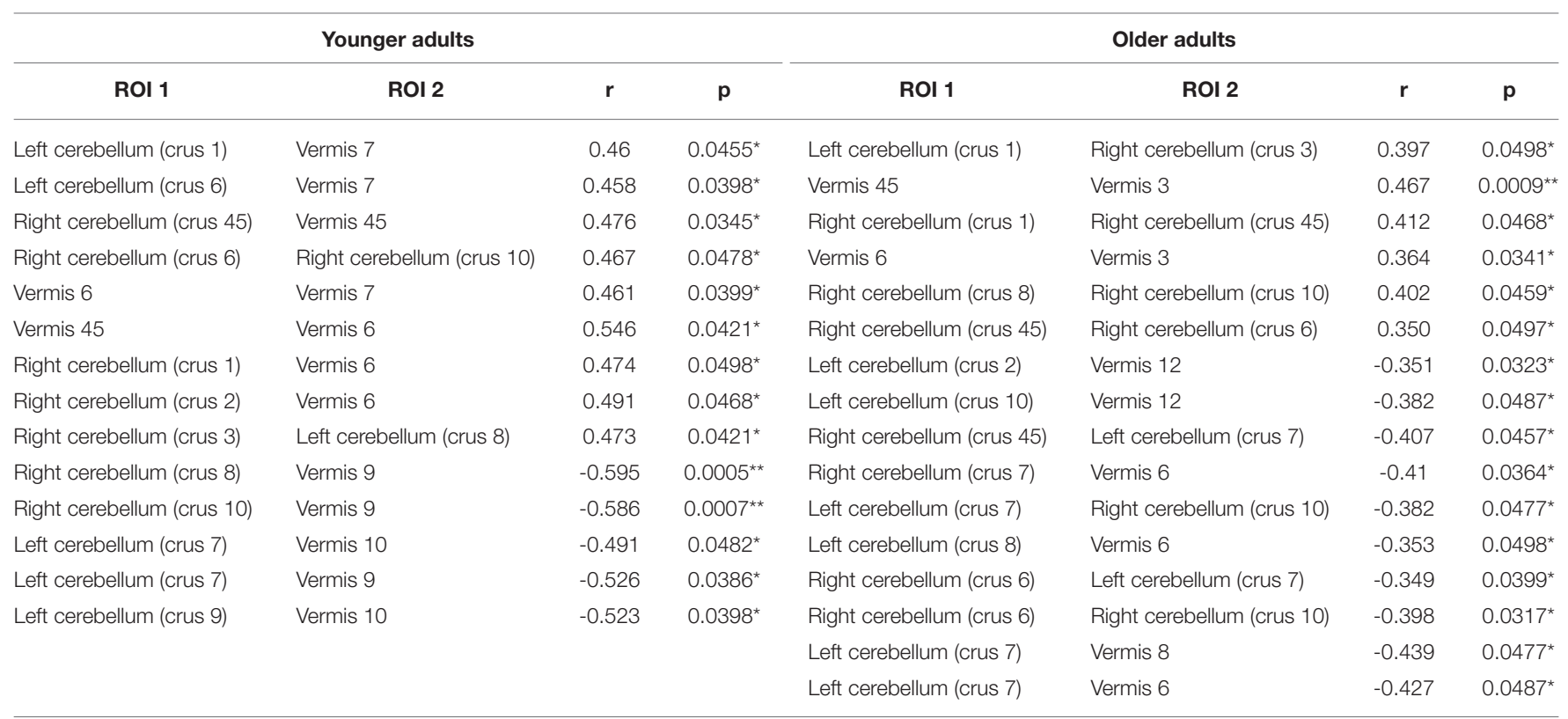

ROI, Region of interest; $r$, Pearson's correlation coefficient; $p$, Significance value. All results were obtained with the threshold significance set to an uncorrected $p<0.001$ at a voxel-wise level, with the false discovery rate (FDR)-corrected $p<0.05$ at the cluster-wise level. ${ }^{*} p<0.05$; ${ }^{* *} p<0.001$. 
The older adults in our study also exhibited stronger cerebellar connectivity between posterior regions compared with the frontal regions, regardless of creative score modulation. In contrast, the younger adults exhibited cerebro-cerebellar connectivity within and between regions of the $\mathrm{CN}$, fronto-parietal network, DMN, $\mathrm{VN}, \mathrm{AN}$, and SMN. The relatively strong connectivity in the posterior regions (vs. frontal regions) of the older adults suggests that a shift in functional connectivity patterns with age could partially support the concept of a posterior-anterior shift in aging (Davis et al., 2008; Zhang et al., 2017).

In our rs-fMRI study, we further examined the critical role of the cerebellum and its distributed connectivity with various regions of the brain associated with creative cognition. Although the cerebellum has traditionally been understood to contribute motor control (Ito, 2000; Ivry et al., 2002; Bastian, 2006), recent studies have suggested that this brain region likely contributes to various complex cognitive tasks (Diedrichsen et al., 2019; Schmahmann, 2019). The cerebellum comprises 69 billion neurons, whereas the cerebral cortex contains only 16 billion neurons (Lent et al., 2012; Lin et al., 2020; Sereno et al., 2020). Consequently, the cerebellum contributes to complex circuitous processes such as sensorimotor function, cognitive functions, and autonomic tasks (Baumann et al., 2015; Schmahmann et al., 2019). The cerebellum has also been shown to contribute to cognitive abilities such as spatial reasoning, language, and working memory (Schmahmann and Sherman, 1998; Ito, 2008; Stoodley and Schmahmann, 2009; Moberget and Ivry, 2016). In addition to anatomical findings, previous rs-fMRI studies have suggested that higher-level cognitive and non-motor functions may be supported by cerebro-cerebellar connections (Habas et al., 2009; Krienen and Buckner, 2009; O'Reilly et al., 2010). Together with evidence from previous rs-fMRI studies that suggested the distribution of CN participation in sensorimotor and higher cognitive functions, our findings suggest that changes in functional connectivity patterns within and between the cerebro-cerebellar circuitry may be critical to understanding the process of complex cognition, including creativity (Akshoomoff et al., 1997; Imamizu et al., 2003; Lin et al., 2020; Sereno et al., 2020).

In our study, CAQ scores exhibited a significant association with RSFC. In older adults, higher connectivity was observed in the superior frontal and posterior regions of the brain, which exhibited significant correlations with individual CAQ scores. In summary, CAQ scores were correlated with regions involving creative cognition in the older participating adults. In contrast, the younger adults exhibited higher connectivity in most of the frontal regions, and this was significantly correlated with CAQ scores. In the cingulate cortex, the older adults exhibited higher connectivity, whereas the younger adults exhibited lower connectivity. Both the older and younger adults also exhibited lower RSFC in these regions, which were significantly correlated with the CAQ scores. We also analyzed functional connectivity in the angular gyrus, which serves as a hub for higher cognitive functions (Fjell et al., 2015) related to episodic and semantic memory, language, and visual, attention, and social cognition (Vincent et al., 2006; Seghier et al., 2010; Price et al., 2015). In the older adults, higher functional connectivity was observed in posterior regions of the angular gyrus (e.g., planum polare, central and frontal operculum), and these correlated with the CAQ scores. In the younger adults, correlations were observed between frontal regions of the angular gyrus (inferior, middle, and superior) and CAQ scores, which indicated higher connectivity within the angular gyrus and particularly the frontal regions. In the fusiform gyrus resting state network, older adults with higher CAQ scores exhibited stronger connectivity in the frontal and parietal regions, and this effect was observed from the posterior to the anterior part of the aging brain. Finally, we explored the contributions of various cerebellar areas to creativity. Although the older adults exhibited smaller increases in cerebellar connectivity than the younger adults, the former group exhibited a greater number of links related to creativity. The observations of significant correlations between the CAQ score and the cerebellum in both older and younger adults emphasize the role of cerebellar resting-state connectivity in creativity.

Some potential limitations of this study must be noted. First, the behavioral data were collected using a standard questionnaire. Future investigations could examine the task-based creative potential of individual participants. Second, the analysis of resting-state connectivity between creative regions in the younger and older participants did not consider individual differences in the CAQ scores or the high and low CAQ scores. Third, the data were collected from a sample of Taiwanese adults, so the generalizability of the results may be limited. Given the previous studies suggested the effects of age on cognitive tasks may be modulated by cultural values (Park and Huang, 2010; $\mathrm{Na}$ et al., 2017), future studies should examine creative cognition in a cross-cultural sample. A few other limitations in our study were the lower sample size and limited statistical power due to 132 ROIs and 11 RSNs. Despite having these limitations, the results have been consistent with prior rs-fMRI creativity works and match with the earlier neuroimaging results and this study identified brain regions and networks that can predict creative styles and achievements and differences in these aspects between younger and older adults.

In conclusion, this combined group-ICA and rs-fMRI study provides network neuroscience based evidence that age-related changes in functional connectivity in the default-executive and cerebro-cerebellar networks modulate the process of creative cognition. Our findings lay a foundation for further studies of how cerebro-cerebellar network interactions may contribute to age-related and individual differences in complex cognitive processes.

\section{DATA AVAILABILITY STATEMENT}

The raw data supporting the conclusions of this article will be made available by the authors, without undue reservation.

\section{ETHICS STATEMENT}

The studies involving human participants were reviewed and approved by The Institutional Review Board of National Chiao 
Tung University, Taiwan. The patients/participants provided their written informed consent to participate in this study.

\section{AUTHOR CONTRIBUTIONS}

$\mathrm{AP}, \mathrm{DM}$, and $\mathrm{C}-\mathrm{MH}$ designed the project. $\mathrm{AP}$ and $\mathrm{C}-\mathrm{MH}$ performed the experiment, analyzed the data, and wrote the manuscript. All authors have read and approved the final version of the manuscript.

\section{FUNDING}

This work was supported by grants from the Academia Sinica Thematic Research Program (AS-103-TP-C04)

\section{REFERENCES}

Abou Elseoud, A., Littow, H., Remes, J., Starck, T., Nikkinen, J., Nissilä, J., et al. (2011). Group-ICA model order highlights patterns of functional brain connectivity. Front. Syst. Neurosci. 5:37. doi: 10.3389/fnsys.2011.00037

Abou-Elseoud, A., Starck, T., Remes, J., Nikkinen, J., Tervonen, O., and Kiviniemi, V. (2010). The effect of model order selection in group PICA. Hum. Brain Mapp. 31, 1207-1216. doi: 10.1002/hbm.20929

Abraham, A., Beudt, S., Ott, D., and Yves von Cramon, D. (2012). Creative cognition and the brain: dissociations between frontal, parietal-temporal and basal ganglia groups. Brain Res. 1482, 55-70. doi: 10.1016/j.brainres.2012.09.007

Addis, D. R., Pan, L., Musicaro, R., and Schacter, D. L. (2016). Divergent thinking and constructing episodic simulations. Memory 24, 89-97. doi: 10.1080/09658211.2014.985591

Adnan, A., Beaty, R., Lam, J., Spreng, R. N., and Turner, G. R. (2019a). Intrinsic default-executive coupling of the creative aging brain. Social Cogn. Affect. Neurosci. 14, 291-303. doi: 10.1093/scan/nsz013

Adnan, A., Beaty, R., Silvia, P., Spreng, R. N., and Turner, G. R. (2019b). Creative aging: functional brain networks associated with divergent thinking in older and younger adults. Neurobiol. Aging 75, 150-158. doi: 10.1016/j.neurobiolaging.2018.11.004

Akshoomoff, N., Courchesne, E., and Townsend, J. (1997). Attention coordination and anticipatory control. Int. Rev. Neurobiol. 41, 575-598. doi: 10.1016/S0074-7742(08)60371-2

Ashburner, J., and Friston, K. (2005). Unified segmentation. Neuroimage 26, 839-851. doi: 10.1016/j.neuroimage.2005.02.018

Bastian, A. J. (2006). Learning to predict the future: the cerebellum adapts feedforward movement control. Curr. Opin. Neurobiol. 16, 645-649. doi: 10.1016/j.conb.2006.08.016

Baumann, O., Borra, R. J., Bower, J. M., Cullen, K. E., Habas, C., Ivry, R. B., et al. (2015). Consensus Paper: the role of the cerebellum in perceptual processes. Cerebellum 14, 197-220. doi: 10.1007/s12311-014-0627-7

Beaty, R. E., Benedek, M., Barry Kaufman, S., and Silvia, P. J. (2015). Default and executive network coupling supports creative idea production. Sci. Rep. 5, 1-14. doi: $10.1038 /$ srep 10964

Beaty, R. E., Benedek, M., Silvia, P. J., and Schacter, D. L. (2016). Creative cognition and brain network dynamics. Trends Cogn. Sci. 20, 87-95. doi: 10.1016/j.tics.2015.10.004

Beaty, R. E., Benedek, M., Wilkins, R. W., Jauk, E., Fink, A., Silvia, P. J., et al. (2014). Creativity and the default network: a functional connectivity analysis of the creative brain at rest. Neuropsychologia 64, 92-98. doi: 10.1016/j.neuropsychologia.2014.09.019

Beaty, R. E., Chen, Q., Christensen, A. P., Qiu, J., Silvia, P. J., and Schacter, D. L. (2018a). Brain networks of the imaginative mind: dynamic functional connectivity of default and cognitive control networks relates to openness to experience. Hum. Brain Mapp. 39, 811-821. doi: 10.1002/hbm.23884 and the Ministry of Science and Technology of Taiwan (105-2420-H-009-001-MY2;107-2410-H-009-028-MY3;108-

2321-B-038-005-MY2) awarded to C-MH. This work was also supported by the Center for Intelligent Drug Systems and Smart Bio-devices (IDS2B) from The Featured Areas Research Center Program within the framework of the Higher Education Sprout Project by the Ministry of Education (MOE) in Taiwan.

\section{ACKNOWLEDGMENTS}

C-MH would like to thank Hsu-Wen Huang and Shih-Ping Huang for their company and indispensable support during the COVID-19 self-quarantine.

Beaty, R. E., Kenett, Y. N., Christensen, A. P., Rosenberg, M. D., Benedek, M., and Chen, Q. (2018b). Robust prediction of individual creative ability from brain functional connectivity. Proc. Natl. Acad. Sci. U.S.A. 115, 1087-1092. doi: $10.1073 /$ pnas. 1713532115

Beaty, R. E., Seli, P., and Schacter, D. L. (2019). Network neuroscience of creative cognition: mapping cognitive mechanisms and individual differences in the creative brain. Curr. Opin. Behav. Sci. 27, 22-30. doi: 10.1016/j.cobeha.2018.08.013

Beckmann, C. F., DeLuca, M., Devlin, J. T., and Smith, S. M. (2005). Investigations into resting-state connectivity using independent component analysis. Philos. Trans. R. Soc. B Biol. Sci. 360, 1001-1013. doi: 10.1098/rstb.2005.1634

Beckmann, C. F., and Smith, S. M. (2005). Tensorial extensions of independent component analysis for multisubject FMRI analysis. Neuroimage 25, 294-311. doi: 10.1016/j.neuroimage.2004.10.043

Behzadi, Y., Restom, K., Liau, J., and Liu, T. T. (2007). A component based noise correction method (CompCor) for BOLD and perfusion based fMRI. Neuroimage 37, 90-101. doi: 10.1016/j.neuroimage.2007.04.042

Benedek, M., Beaty, R., Jauk, E., Koschutnig, K., Fink, A., Silvia, P. J., et al. (2014a). Creating metaphors: the neural basis of figurative language production. Neuroimage 90, 99-106. doi: 10.1016/j.neuroimage.2013.12.046

Benedek, M., Jauk, E., Beaty, R. E., Fink, A., Koschutnig, K., and Neubauer, A. C. (2016). Brain mechanisms associated with internally directed attention and self-generated thought. Sci. Rep. 6:22959. doi: 10.1038/srep22959

Benedek, M., Jauk, E., Fink, A., Koschutnig, K., Reishofer, G., Ebner, F., et al. (2014b). To create or to recall? Neural mechanisms underlying the generation of creative new ideas. Neuroimage 88, 125-133. doi: 10.1016/j.neuroimage.2013.11.021

Benedek, M., Jauk, E., Sommer, M., Arendasy, M., and Neubauer, A. C. (2014c). Intelligence, creativity, and cognitive control: the common and differential involvement of executive functions in intelligence and creativity. Intelligence 46, 73-83. doi: 10.1016/j.intell.2014.05.007

Bernard, J. A., and Seidler, R. D. (2014). Moving forward: age effects on the cerebellum underlie cognitive and motor declines. Neurosci. Biobehav. Rev. 42, 193-207. doi: 10.1016/j.neubiorev.2014.02.011

Bhaganagarapu, K., Jackson, G. D., and Abbott, D. F. (2013). An automated method for identifying artifact in independent component analysis of resting-state fMRI. Front. Hum. Neurosci. 7:343. doi: 10.3389/fnhum.201 3.00343

Bi, X. A., Sun, Q., Zhao, J., Xu, Q., and Wang, L. (2018). Non-linear ICA analysis of resting-state fMRI in mild cognitive impairment. Front. Neurosci. 12:413. doi: 10.3389/fnins.2018.00413

Bressler, S. L., and Menon, V. (2010). Large-scale brain networks in cognition: emerging methods and principles. Trends Cogn. Sci. 14, 277-290. doi: 10.1016/j.tics.2010.04.004

Buckner, R. L. (2013). The cerebellum and cognitive function: 25 years of insight from anatomy and neuroimaging. Neuron 80, 807-815. doi: 10.1016/j.neuron.2013.10.044 
Buckner, R. L., and DiNicola, L. M. (2019). The brain's default network: updated anatomy, physiology and evolving insights. Nat. Rev. Neurosci. 20, 593-608. doi: 10.1038/s41583-019-0212-7

Calhoun, V. D., and Adali, T. (2006). Unmixing fMRI with independent component analysis. IEEE Eng. Med. Biol. Mag. 25, 79-90. doi: 10.1109/MEMB.2006.1607672

Calhoun, V. D., Adali, T., Pearlson, G. D., and Pekar, J. J. (2001). A method for making group inferences from functional MRI data using independent component analysis. Hum. Brain Mapp. 14, 140-151. doi: 10.1002/hbm.1048

Carson, S., Peterson, J. B., and Higgins, D. M. (2005). Reliability, validity, and factor structure of the creative achievement questionnaire. Creat. Res. J. 17, 37-50. doi: 10.1207/s15326934crj1701_4

Chai, X. J., Ofen, N., Gabrieli, J. D. E., and Whitfield-Gabrieli, S. (2014). Development of deactivation of the default-mode network during episodic memory formation. Neuroimage 84, 932-938. doi: 10.1016/j.neuroimage.2013.09.032

Chen, Q., Beaty, R. E., Cui, Z., Sun, J., He, H., Zhuang, K., et al. (2019). Brain hemispheric involvement in visuospatial and verbal divergent thinking. Neuroimage 202:116065. doi: 10.1016/j.neuroimage.2019.116065

Cole, D. M., Smith, S. M., and Beckmann, C. F. (2010). Advances and pitfalls in the analysis and interpretation of resting-state FMRI data. Front. Syst. Neurosci. 4:8. doi: 10.3389/fnsys.2010.00008

Davis, S. W., Dennis, N. A., Daselaar, S. M., Fleck, M. S., and Cabeza, R. (2008). Que PASA? The posterior-anterior shift in aging. Cereb. Cortex 18, 1201-1209. doi: 10.1093/cercor/bhm155

den Heuvel, O. A., der Werf, Y. D., Verhoef, K. M., de Wit, S., Berendse, H. W., Wolters, E. C., et al. (2010). Frontal-striatal abnormalities underlying behaviours in the compulsive-impulsive spectrum. J. Neurol. Sci. 289, 55-59. doi: 10.1016/j.jns.2009.08.043

Diedrichsen, J., King, M., Hernandez-Castillo, C., Sereno, M., and Ivry, R. B. (2019). Universal transform or multiple functionality? Understanding the contribution of the human cerebellum across task domains. Neuron 102, 918-928. doi: 10.1016/j.neuron.2019.04.021

Dietrich, A., and Kanso, R. (2010). A review of EEG, ERP, and neuroimaging studies of creativity and insight. Psychol. Bull. 136, 822-848. doi: $10.1037 / \mathrm{a} 0019749$

Duff, M. C., Kurczek, J., Rubin, R., Cohen, N. J., and Tranel, D. (2013). Hippocampal amnesia disturb creative thinking. Hippocampus 1, 1143-1149. doi: $10.1002 /$ hipo. 22208

Erhardt, E. B., Rachakonda, S., Bedrick, E. J., Allen, E. A., Adali, T., and Calhoun, V. D. (2011). Comparison of multi-subject ICA methods for analysis of fMRI data. Hum. Brain Mapp. 32, 2075-2095. doi: 10.1002/hbm.21170

Fan, Y.-T., Fang, Y.-W., Chen, Y.-P., Leshikar, E. D., Lin, C.-P., Tzeng, O. J. L., et al. (2019). Aging, cognition, and the brain: effects of age-related variation in white matter integrity on neuropsychological function. Aging Mental Health 23, 831-839. doi: 10.1080/13607863.2018.1455804

Feng, Q., He, L., Yang, W., Zhang, Y., Wu, X., and Qiu, J. (2019). Verbal creativity is correlated with the dynamic reconfiguration of brain networks in the resting state. Front. Psychol. 10:894. doi: 10.3389/fpsyg.2019.00894

Fink, A., Grabner, R. H., Benedek, M., Reishofer, G., Hauswirth, V., Fally, M., et al. (2009). The creative brain: Investigation of brain activity during creative problem solving by means of EEG and fMRI. Hum. Brain Mapp. 748, 734-748. doi: 10.1002/hbm.20538

Fink, A., Grabner, R. H., Gebauer, D., Reishofer, G., Koschutnig, K., and Ebner, F. (2010). Enhancing creativity by means of cognitive stimulation: Evidence from an fMRI study. Neuroimage 52, 1687-1695. doi: 10.1016/j.neuroimage.2010.05.072

Fink, A., Weber, B., Koschutnig, K., Benedek, M., Reishofer, G., Ebner, F., et al. (2014). Creativity and schizotypy from the neuroscience perspective. Cogn. Affect. Behav. Neurosci. 14, 378-387. doi: 10.3758/s13415-013-0210-6

Fjell, A. M., Westlye, L. T., Amlien, I., Tamnes, C. K., Grydeland, H., Engvig, A., et al. (2015). High-expanding cortical regions in human development and evolution are related to higher intellectual abilities. Cereb. Cortex 25, 26-34. doi: 10.1093/cercor/bht201

Folstein, M. F., Folstein, S. E., and McHugh, P. R. (1975). "Mini-mental state". A practical method for grading the cognitive state of patients for the clinician. J. Psychiatr. Res. 12, 189-198. doi: 10.1016/0022-3956(75)9 0026-6
Foos, P., and Boone, D. (2008). Adult age differences in divergent thinking: it's just a matter of time. Educ. Gerontol. 34, 587-594. doi: 10.1080/03601270801949393

Fox, M. D., and Raichle, M. E. (2007). Spontaneous fluctuations in brain activity observed with functional magnetic resonance imaging. Nat. Rev. Neurosci. 8, 700-711. doi: 10.1038/nrn2201

Geissmann, L., Gschwind, L., Schicktanz, N., Deuring, G., Rosburg, T., Schwegler, K., et al. (2018). Resting-state functional connectivity remains unaffected by preceding exposure to aversive visual stimuli. Neuroimage 167, 354-365. doi: 10.1016/j.neuroimage.2017.11.046

Green, A. E., Cohen, M. S., Raab, H. A., Yedibalian, C. G., and Gray, J. R. (2015). Frontopolar activity and connectivity support dynamic conscious augmentation of creative state. Hum. Brain Mapp. 36, 923-934. doi: 10.1002/hbm.22676

Habas, C., Kamdar, N., Nguyen, D., Prater, K., Beckmann, C. F., Menon, V., et al. (2009). Distinct cerebellar contributions to intrinsic connectivity networks. J. Neurosci. 29, 8586-8594. doi: 10.1523/JNEUROSCI.1868-09.2009

Honda, T., Nagao, S., Hashimoto, Y., Ishikawa, K., Yokota, T., Mizusawa, H., et al. (2018). Tandem internal models execute motor learning in the cerebellum. Proc. Natl. Acad. Sci. U.S.A. 115, 7428-7433. doi: 10.1073/pnas.1716489115

Huang, C.-M., Polk, T. A., Goh, J. O., and Park, D. C. (2012). Both left and right posterior parietal activations contribute to compensatory processes in normal aging. Neuropsychologia 50, 55-66. doi: 10.1016/j.neuropsychologia.2011.10.022

Imamizu, H., Kuroda, T., Miyauchi, S., Yoshioka, T., and Kawato, M. (2003). Modular organization of internal models of tools in the human cerebellum. Proc. Natl. Acad. Sci. U.S.A. 100, 5461-5466. doi: 10.1073/pnas.0835746100

Ito, M. (1993). Movement and thought: Identical control mechanisms by the cerebellum. Trends Neurosci. 16, 448-450. doi: 10.1016/0166-2236(93)90073-U

Ito, M. (2000). Mechanisms of motor learning in the cerebellum. Brain Res. 886, 237-245. doi: 10.1016/S0006-8993(00)03142-5

Ito, M. (2006). Cerebellar circuitry as a neuronal machine. Prog. Neurobiol. 78, 272-303. doi: 10.1016/j.pneurobio.2006.02.006

Ito, M. (2008). Control of mental activities by internal models in the cerebellum. Nat. Rev. Neurosci. 9, 304-313. doi: 10.1038/nrn2332

Ivry, R. B., Spencer, R. M., Zelaznik, H. N., and Diedrichsen, J. (2002). The cerebellum and event timing. Ann. N. Y. Acad. Sci. 978, 302-317. doi: 10.1111/j.1749-6632.2002.tb07576.x

Jauk, E., Benedek, M., Dunst, B., and Neubauer, A. C. (2013). The relationship between intelligence and creativity: New support for the threshold hypothesis by means of empirical breakpoint detection. Intelligence 41, 212-221. doi: 10.1016/j.intell.2013.03.003

Jung, R. E., Mead, B. S., Carrasco, J., and Flores, R. A. (2013). The structure of creative cognition in the human brain. Hum. Neurosci. 7, 1-13. doi: 10.3389/fnhum.2013.00330

Jung, R. E., Segall, J. M., Bockholt, H. J., Flores, R. A., Smith, S. M., Chavez, R. S., et al. (2010). Neuroanatomy of creativity. Hum. Brain Mapp. 31, 398-409. doi: $10.1002 / \mathrm{hbm} .20874$

Kenett, Y. N., Anaki, D., and Faust, M. (2014). Investigating the structure of semantic networks in low and high creative persons. Front. Hum. Neurosci. 8:407. doi: 10.3389/fnhum.2014.00407

Kenett, Y. N., Anaki, D., and Faust, M. (2015). Processing of unconventional stimuli requires the recruitment of the non-specialized hemisphere. Front. Hum. Neurosci. 9:32. doi: 10.3389/fnhum.2015.00032

Kornelsen, J., Wilson, A., Labus, J. S., Witges, K., Mayer, E. A., and Bernstein, C. N. (2020). Brain resting-state network alterations associated with Crohn's disease. Front. Neurol. 11:48. doi: 10.3389/fneur.2020.00048

Krienen, F. M., and Buckner, R. L. (2009). Segregated fronto-cerebellar circuits revealed by intrinsic functional connectivity. Cereb. Cortex 19, 2485-2497. doi: 10.1093/cercor/bhp135

Lent, R., Azevedo, F. A., Andrade-Moraes, C. H., and Pinto, A. V. (2012). How many neurons do you have? Some dogmas of quantitative neuroscience under revision. Eur. J. Neurosci. 35, 1-9. doi: 10.1111/j.1460-9568.2011.07923.x

Li, Y.-O., Adali, T., and Calhoun, V. D. (2007). Estimating the number of independent components for functional magnetic resonance imaging data. Hum. Brain Mapp. 28, 1251-1266. doi: 10.1002/hbm.20359

Lin, Q., Manley, J., Helmreich, M., Schlumm, F., Li, J. M., Robson, D. N., et al. (2020). Cerebellar neurodynamics predict decision timing and outcome on the single-trial level. Cell 180, 536-551. doi: 10.1016/j.cell.2019.12.018 
Liu, S., Erkkinen, M. G., Healey, M. L., Xu, Y., Swett, K. E., Chow, H. M., et al. (2015). Brain activity and connectivity during poetry composition: toward a multidimensional model of the creative process. Hum. Brain Mapp. 36, 3351-3372. doi: 10.1002/hbm.22849

Mammarella, I. C., Giofré, D., Caviola, S., Cornoldi, C., and Hamilton, C. (2014). Visuospatial working memory in children with autism: The effect of a semantic global organization. Res. Dev. Disabil. 35, 1349-1356. doi: 10.1016/j.ridd.2014.03.030

Manoliu, A., Riedl, V., Doll, A., Bäuml, J. G., Mühlau, M., Schwerthöffer, D., et al. (2013). Insular dysfunction reflects altered between-network connectivity and severity of negative symptoms in schizophrenia during psychotic remission. Front. Hum. Neurosci. 7:216. doi: 10.3389/fnhum.2013.00216

Markman, A., and Dietrich, E. (2010). Extending the classical view of representation. Trends Cogn. Sci. 4:470. doi: 10.1016/S1364-6613(00)01559-X

McKeown, M. J., Jung, T.-P., Bell, A. J., Sejnowski, T. J., Makeig, S., Brown, G. G., et al. (1998). Analysis of fMRI data by blind separation into independent spatial components. Hum. Brain Mapp. 6, 160-188. doi: 10.1002/(SICI)1097-0193(1998)6:3<160::AID-HBM5>3.0.CO;2-1

Moberget, T., and Ivry, R. B. (2016). Cerebellar contributions to motor control and language comprehension: searching for common computational principles. Ann. N. Y. Acad. Sci. 1369, 154-171. doi: 10.1111/nyas.13094

Na, J., Huang, C.-M., and Park, D. C. (2017). When age and culture interact in an easy and yet cognitively demanding task: older adults, but not younger adults, showed the expected cultural differences. Front. Psychol. 8:457. doi: 10.3389/fpsyg.2017.00457

Neumann, N., Domin, M., Erhard, K., and Lotze, M. (2018). Voxel-based morphometry in creative writers: grey matter increase in a prefronto-thalamiccerebellar network. Eur. J. Neurosci. 48, 1647-1653. doi: 10.1111/ejn.13952

Ogawa, T., Aihara, T., Shimokawa, T., and Yamashita, O. (2018). Largescale brain network associated with creative insight: Combined voxel-based morphometry and resting-state functional connectivity analyses. Sci. Rep. 8, 1-11. doi: 10.1038/s41598-018-24981-0

O’Reilly, J. X., Beckmann, C. F., Tomassini, V., Ramnani, N., and JohansenBerg, H. (2010). Distinct and overlapping functional zones in the cerebellum defined by resting state functional connectivity. Cereb. Cortex 20, 953-965. doi: 10.1093/cercor/bhp157

Palmiero, M., Di Giacomo, D., and Passafiume, D. (2014). Divergent thinking and age-related changes. Creat. Res. J. 26, 456-460. doi: 10.1080/10400419.2014.961786

Park, D. C., and Huang, C.-M. (2010). Culture wires the brain: a cognitive neuroscience perspective. Perspect. Psychol. Sci. 5, 391-400. doi: $10.1177 / 1745691610374591$

Park, D. C., Lautenschlager, G., Hedden, T., Davidson, N. S., Smith, A. D., and Smith, P. K. (2002). Models of visuospatial and verbal memory across the adult life span. Psychol. Aging 17, 299-320. doi: 10.1037/0882-7974.17.2.299

Pinho, A. L., de Manzano, R., Fransson, P., Eriksson, H., and Ullén, F. (2014). Connecting to create: expertise in musical improvisation is associated with increased functional connectivity between premotor and prefrontal areas. $J$. Neurosci. 34, 6156-6163. doi: 10.1523/JNEUROSCI.4769-13.2014

Price, A. R., Bonner, M. F., Peelle, J. E., and Grossman, M. (2015). Converging evidence for the neuroanatomic basis of combinatorial semantics in the angular gyrus. J. Neurosci. 35, 3276-3284. doi: 10.1523/JNEUROSCI.3446-14.2015

Raichle, M. E. (2010). Two views of brain function. Trends Cogn. Sci. 14, 180-190. doi: $10.1016 /$ j.tics.2010.01.008

Ramnani, N. (2006). The primate cortico-cerebellar system: anatomy and function. Nat. Rev. Neurosci. 7, 511-522. doi: 10.1038/nrn1953

Raz, N., Lindenberger, U., Rodrigue, K. M., Kennedy, K. M., Head, D., Williamson, A., et al. (2005). Regional brain changes in aging healthy adults: general trends, individual differences and modifiers. Cereb. Cortex 15, 1676-1689. doi: $10.1093 /$ cercor/bhi044

Reese, H. W., Lee, L. J., Cohen, S. H., and Puckett, J. M. (2001). Effects intellectual variables, ages, and gender on divergent thinking in adulthood. Int. J. Behav. Dev. 25, 491-500. doi: 10.1080/01650250042000483

Roskos-Ewoldsen, B., Black, S. R., and Mccown, S. M. (2008). Agerelated changes in creative thinking. J. Creat. Behav. 42, 33-59. doi: 10.1002/j.2162-6057.2008.tb01079.x

Rummel, C., Verma, R. K., Schöpf, V., Abela, E., Hauf, M., Berruecos, J. F. Z., et al. (2013). Time course based artifact identification for independent components of resting-state fMRI. Front. Hum. Neurosci. 7:214. doi: 10.3389/fnhum.2013.00214

Saggar, M., Quintin, E. M., Kienitz, E., Bott, N. T., Sun, Z., Hong, W. C., et al. (2015). Pictionary-based fMRI paradigm to study the neural correlates of spontaneous improvisation and figural creativity. Sci. Rep. 5, 1-11. doi: $10.1038 /$ srep 10894

Schmahmann, J. D. (1991). An emerging concept the cerebellar contribution to higher function. Neurol. Rev. 48, 1178-1187. doi: 10.1001/archneur.1991.00530230086029

Schmahmann, J. D. (2019). The cerebellum and cognition. Neurosci. Lett. 688, 62-75. doi: 10.1016/j.neulet.2018.07.005

Schmahmann, J. D., Guell, X., Stoodley, C. J., and Halko, M. A. (2019). The theory and neuroscience of cerebellar cognition. Annu. Rev. Neurosci. 42, 337-364. doi: 10.1146/annurev-neuro-070918-050258

Schmahmann, J. D., and Sherman, J. C. (1998). The cerebellar cognitive affective syndrome. Brain 121, 561-579. doi: 10.1093/brain/121.4.561

Seghier, M. L., Fagan, E., and Price, C. J. (2010). Functional subdivisions in the left angular gyrus where the semantic system meets and diverges from the default network. J. Neurosci. 30, 16809-16817. doi: 10.1523/JNEUROSCI.3377-10.2010

Sereno, M. I., Diedrichsen, J., Tachrount, M., Testa-Silva, G., Arceuil, H., and De Zeeuw, C. (2020). The human cerebellum has almost $80 \%$ of the surface area of the neocortex. Proc. Natl. Acad. Sci. U.S.A. 117, 19538-19543. doi: 10.1073/pnas.2002896117

Silvia, P. J., Beaty, R. E., and Nusbaum, E. C. (2013). Verbal fluency and creativity: general and specific contributions of broad retrieval ability (Gr) factors to divergent thinking. Intelligence 41, 328-340. doi: 10.1016/j.intell.2013.05.004

Silvia, P. J., Kaufman, J. C., and Pretz, J. E. (2009). Is Creativity domainspecific? Latent class models of Creative accomplishments and Creative selfdescriptions. Psychol. Aesthet. Creat Arts 3, 139-148. doi: 10.1037/a0014940

Spreng, R. N., Jorge, S., Turner, G. R., Stevens, W. D., and Schacter, D. L. (2013). Intrinsic architecture underlying the relations among the default, dorsal attention, and frontoparietal control networks of the human brain. J. Cogn. Neurosci. 25, 1-10. doi: 10.1162/jocn_a_00281

Spreng, R. N., and Turner, G. R. (2019). The shifting architecture of cognition and brain function in older adulthood. Perspect. Psychol. Sci. 14, 523-542. doi: $10.1177 / 1745691619827511$

Stoodley, C. J., and Schmahmann, J. D. (2009). Functional topography in the human cerebellum: a meta-analysis of neuroimaging studies. Neuroimage 44, 489-501. doi: 10.1016/j.neuroimage.2008.08.039

Stoodley, C. J., Valera, E., and Schmahmann, J. (2012). Functional topography of the cerebellum for motor and cognitive tasks: an fMRI study. Neuroimage 59, 1560-1570. doi: 10.1016/j.neuroimage.2011.08.065

Sun, J., Liu, Z., Rolls, E. T., Chen, Q., Yao, Y., Yang, W., et al. (2019). Verbal creativity correlates with the temporal variability of brain networks during the resting state. Cereb. Cortex 29, 1047-1058. doi: 10.1093/cercor/bhy010

Sunavsky, A., and Poppenk, J. (2019). Neuroimaging predictors of creativity in healthy adults. Neuroimage 206:116292. doi: 10.1016/j.neuroimage.2019.116292

Takeuchi, H., Taki, Y., Hashizume, H., Sassa, Y., Nagase, T., Nouchi, R., et al. (2011). Failing to deactivate: the association between brain activity during a working memory task and creativity. Neuroimage 55, 681-687. doi: 10.1016/j.neuroimage.2010.11.052

Takeuchi, H., Taki, Y., Hashizume, H., Sassa, Y., Nagase, T., Nouchi, R., et al. (2012). The association between resting functional connectivity and creativity. Sci. Rep. 6, 2921-2929. doi: 10.1093/cercor/bhr371

Turner, G. R., and Spreng, R. N. (2015). Prefrontal engagement and reduced default network suppression co-occur and are dynamically coupled in older adults: the default-executive coupling hypothesis of aging. J. Cogn. Neurosci. 27, 2462-2476. doi: 10.1162/jocn_a_00869

Tzourio-Mazoyer, N., Landeau, B., Papathanassiou, D., Crivello, F., Etard, O., Delcroix, N., et al. (2002). Automated anatomical labeling of activations in SPM using a macroscopic anatomical parcellation of the MNI MRI single-subject brain. Neuroimage 15, 273-289. doi: 10.1006/nimg.2001.0978

van den Heuvel, M. P., and Hulshoff Pol, H. E. (2010). Exploring the brain network: a review on resting-state fMRI functional connectivity. Eur. Neuropsychopharmacol. 20, 519-534. doi: 10.1016/j.euroneuro.2010.03.008

Vandervert, L. R., Schimpf, P. H., Liu, H., Vandervert, L. R., Schimpf, P. H., and Liu, H. (2007). How working memory and the cerebellum 
collaborate to produce creativity and innovation. Creat. Res. J. 19, 1-18. doi: 10.1080/10400410709336873

Vincent, J. L., Snyder, A. Z., Fox, M. D., Shannon, B., Andrews, J., Raichle, M. E., et al. (2006). Coherent spontaneous activity identifies a hippocampal-parietal memory network. J. Neurophysiol. 96, 3517-3531. doi: 10.1152/jn.00048.2006

Wechsler, D. (1997a). Wechsler Adult Intelligence Scale, 3rd Edn. San Antonio, TX: The Psychological Corporation. doi: 10.1037/t49755-000

Wechsler, D. (1997b). Wechsler Memory Scale, 3rd Edn. San Antonio, TX: The Psychological Corporation.

Wei, D., Yang, J., Li, W., Wang, K., Zhang, Q., and Qiu, J. (2014). Increased resting functional connectivity of the medial prefrontal cortex in creativity by means of cognitive stimulation. Cortex 51, 92-102. doi: 10.1016/j.cortex.2013.09.004

Wertz, C. J., Chohan, M. O., Flores, R. A., and Jung, R.ex, E. (2020). Neuroanatomy of creative achievement. Neuroimage 209:116487. doi: 10.1016/j.neuroimage.2019.116487

Whitfield-Gabrieli, S., and Nieto-Castanon, A. (2012). Conn: a functional connectivity toolbox for correlated and anticorrelated brain networks. Brain Connect. 2, 125-141. doi: 10.1089/brain.20 12.0073

Zhang, H., Lee, A., and Qiu, A. (2017). A posterior-to-anterior shift of brain functional dynamics in aging. Brain Struct. Funct. 222, 3665-3676. doi: $10.1007 /$ s00429-017-1425-Z

Conflict of Interest: The authors declare that the research was conducted in the absence of any commercial or financial relationships that could be construed as a potential conflict of interest.

Copyright (c) 2021 Patil, Madathil and Huang. This is an open-access article distributed under the terms of the Creative Commons Attribution License (CC BY). The use, distribution or reproduction in other forums is permitted, provided the original author(s) and the copyright owner(s) are credited and that the original publication in this journal is cited, in accordance with accepted academic practice. No use, distribution or reproduction is permitted which does not comply with these terms. 